\title{
Estudo da geração de viagens em instituições de ensino médio
}

\author{
Ângela Beatriz Souza Bertazzo; ${ }^{1}$ Maria Alice Prudêncio Jacques²
}

\begin{abstract}
Resumo: As Instituições de Ensino Médio (IEMs) são Polos Geradores de Viagens (PGVs) e, por isso, a implantação desses empreendimentos requer estudos sobre o seu impacto nos sistemas de transporte e tráfego na área de sua localização. Embora relevante, este tema não tem sido suficientemente explorado na literatura técnica nacional, e as taxas de geração de viagens disponíveis em referências internacionais relacionam-se a IEMs com características, em geral, diferentes das observadas nas instituições brasileiras. Este trabalho tem, portanto, o objetivo de estudar a geração de viagens de IEMs brasileiras, públicas e privadas, visando definir taxas e modelos por modo de transporte para esse tipo de PGV. Os resultados obtidos para IEMs de Brasília apontam, entre outros aspectos, diferenças significativas entre a geração de viagens das IEMs públicas e privadas. A metodologia adotada, baseada na aplicação de questionários a gestores e usuários deste tipo de PGV, pode ser aplicada em outras cidades.
\end{abstract}

\begin{abstract}
High schools are trip generators (PGVs) and, therefore, new high school enterprises require studies on their impact on related traffic and transportation systems. Although important, this issue has not been sufficiently explored in the Brazilian technical literature. In addition, international studies on high schools' travel patterns and trip generation rates consider institutions with characteristics, in general, different from Brazilian schools. In light of this, this work aims to study the trip generation of public and private Brazilian high schools, towards defining trip generation rates and models for this type of PGV, by transportation mode. The results presented by institutions located in Brasilia show, among other aspects, significant differences between the travel patterns, rates and models developed for public and private high schools. The methodology used in this study was based on questionnaires applied to staff and users of this type of PGV, and may be adopted in other cities.
\end{abstract}

\section{INTRODUÇÃO}

A visão tradicional adotada pelos municípios brasileiros é de classificar a necessidade de estudos mais detalhados relacionados à implantação de empreendimentos em áreas urbanas em função das respectivas áreas construídas (Nunes, 2005). Entretanto, o estudo de impacto de novos empreendimentos, especialmente dos classificados como Polos Geradores de Viagens (PGVs), tem evoluído, passando a considerar outros elementos tais como impactos produzidos na acessibilidade e circulação de pedestres nas áreas lindeiras, desenvolvimento socioeconômico local, além de características específicas da circulação de veículos nas áreas internas e externas aos empreendimentos.

O Ministério da Justiça (DENATRAN, 2000), incentivando a implantação de medidas de segurança em áreas escolares, e acompanhando a literatura internacional, salienta que todas as escolas devam ser tratadas como "Micro-polos Geradores de Tráfego". São diversos os problemas verificados junto a esses estabelecimentos, principalmente quanto à falta de inte-

\footnotetext{
${ }^{1}$ Ângela Beatriz Souza Bertazzo, Programa de Pós-Graduação em Transportes, Departamento de Engenharia Civil e Ambiental, Universidade de Brasília, Brasília, DF, Brasil. (e-mail: angela.bertazzo@hotmail.com).

${ }^{2}$ Maria Alice Prudêncio Jacques, Programa de Pós-Graduação em Transportes, Departamento de Engenharia Civil e Ambiental, Universidade de Brasília, Brasília, DF, Brasil. (e-mail: mapj@unb.br).
}

Manuscrito recebido em 9/10/2009 e aprovado para publicação em 25/2/2010. Este artigo é parte de TRANSPORTES, volume XVIII, número 2, junho de 2010. ISSN: 2237-1346 (online). gração do planejamento urbano e do planejamento de transportes. Observa-se que esses conflitos ocorrem principalmente junto aos locais de entrada e saída, pela desconsideração da segurança nos deslocamentos de escolares a pé e priorização da circulação de veículos.

Em qualquer metodologia de estudo de impacto a ser utilizada para analisar a implantação de empreendimentos tipo Polo Gerador de Viagens - PGV, o fundamental é que se tenha de antemão dados que indiquem a capacidade desse tipo de empreendimento em gerar (atrair e produzir) viagens (Portugal e Goldner, 2003). A demanda por viagens é derivada do tipo de atividade desenvolvida no empreendimento e, assim, o estudo de geração de viagens deve ser adaptado para diferentes tipos de atividades.

No que diz respeito às instituições de ensino médio (IEMs), os estudos disponíveis na literatura nacional referentes a taxas e modelos de geração de viagens são bastante reduzidos, cabendo destacar os realizados pela Companhia de Engenharia de Tráfego de São Paulo (CET-SP). Quanto a estudos realizados em outros países, dentre os quais os mais difundidos em nível internacional são os realizados pelo Institute of Transportation Engineers (ITE), cabe levar em conta as diferenças consideráveis entre as características das IEMs americanas e brasileiras.

Neste contexto, o presente trabalho apresenta uma metodologia para estudo dos padrões de viagem dos usuários de instituições de ensino médio. Aplicada a escolas na cidade de Brasília, teve como objetivo a de- 
terminação de taxas e modelos de geração de viagens diferenciadas por caráter de escola, se pública ou privada, e servindo para futuros estudos ligados à análise do impacto deste tipo de empreendimento pelos órgãos gestores de trânsito das cidades brasileiras.

$\mathrm{O}$ artigo aborda, inicialmente, as principais diferenças entre as características das IEMs brasileiras e as das escolas congêneres de outros países, que afetam a realização de estudos de geração de viagens e o uso, no Brasil, de resultados obtidos em outros países. Em seguida, apresenta uma revisão dos métodos para estudos de geração de viagens para IEMs, referidos na literatura nacional e estrangeira, e a metodologia adotada no desenvolvimento do presente estudo. Finalmente, são apresentados os resultados obtidos para a cidade de Brasília, e as conclusões gerais e específicas do trabalho.

\section{INSTITUIÇÕES DE ENSINO MÉDIO E ESTUDOS DE GERAÇÃO DE VIAGENS}

Quanto à determinação de taxas e modelos de viagens, algumas características observadas nas IEMs brasileiras mostram-se divergentes com relação às escolas estudadas em trabalhos realizados no exterior para este tipo de empreendimento. Primeiramente o tipo de gestão da matrícula por vizinhança, ou base territorial, em escolas públicas é muito mais eficaz em países como Estados Unidos, Reino Unido, Japão e Canadá, do que no Brasil.

No que se refere ao acesso à rede de transporte público urbano, no caso brasileiro, esta condição pode ser tão ou mais importante que a presença de áreas de embarque e desembarque para automóveis, pois as escolas brasileiras possuem uma matriz modal diversificada segundo o tipo de IEM, pública ou privada, com forte dependência do transporte público no caso das escolas públicas (Ferreira, 2007). Diferentemente do que ocorre nas IEMs americanas, algumas características observadas das IEMs brasileiras se constituem restrições à aplicação direta das taxas e modelos de geração de viagens do ITE Dentre elas, destaca-se o uso intensivo do transporte público por alunos das escolas públicas de ensino médio, aliado ao fato de que, no Brasil, apenas maiores de 18 anos são habilitados a dirigir automóveis.

$\mathrm{O}$ próprio uso da metodologia do ITE, que requer contagens veiculares junto às instituições para a realização de estudos de geração de viagens, para o caso das IEMs também apresenta limitações para utilização no caso brasileiro. As operações de embarque e desembarque de automóveis nem sempre acontecem de forma contida, em baias ou alças específicas para esse fim, proporcionando a contagem controlada e específica de veículos prevista na metodologia do ITE. No caso brasileiro, tais operações acontecem em geral numa área próxima aos acessos da escola, mas com caráter disperso, segundo a conveniência do motorista e nem sempre considerada a segurança do pedestre. Estas viagens também podem envolver escolares de diferentes graus no mesmo veículo, ou carona entre diferentes tipos de usuários da mesma instituição. Isso porque são poucas as instituições que oferecem exclusivamente o nível médio de ensino. A maior parte integra a oferta de diferentes níveis na mesma escola, otimizando a estrutura física existente, quer num mesmo turno ou em turnos diferentes. Assim, em muitos casos, cada turno pode caracterizar uma população usuária diferente.

A maioria das viagens geradas pelas IEMS, do mesmo modo que ocorre com as demais instituições de ensino, gera viagens regulares em dias úteis. Esta regularidade afeta a determinação de quais serão as viagens veiculares que poderão ser atribuídas efetivamente à implantação do novo empreendimento. Para Souza (2007), a classificação tradicional de viagens primárias, tradicionalmente referidas às viagens de base domiciliar e com destino ou origem no PGV, viagens desviadas e de passagem deve ser adaptada para as instituições de ensino. As viagens denominadas por Souza (2007) como "específicas" (viagens realizadas em função da instituição de ensino, com ou sem base domiciliar) e as viagens desviadas devem ser atribuídas ao impacto do novo empreendimento. Este conceito é empregado no presente trabalho. Além disso, o período de realização preponderante das viagens geradas pelas instituições de ensino ocorre no início e término de cada turno de aulas.

\section{TAXAS E MODELOS DE GERAÇÃO DE VIAGENS PARA IEMS}

Desde os anos de 1980, o ITE vem desenvolvendo e reunindo estudos de geração de viagens para os mais variados tipos de uso de solo, e ainda é a referência mais utilizada para análise de geração de viagens em PGVs. No caso de Instituições de Ensino Médio (High Schools), estão disponíveis nas publicações do ITE taxas médias de viagens para usuários de IEMs (alunos, professores e funcionários) pelas variáveis 'número de alunos', 'número de empregados' e ‘área em 1000 pés quadrados'. As Tabelas 1 e 2 apresentam as taxas elaboradas pelo ITE para dias da semana típicos para as variáveis explicativas "número de alunos" e "número de empregados", respectivamente. Estas taxas foram obtidas a partir de estudos realizados em escolas localizadas nos EUA. Outros resultados disponíveis no ITE (2003), referentes a viagens em fins de semana e relativas à variável explicativa "1000 pés quadrados de área", não obtiveram resultados significativos e não 
Tabela 1. Resultados ITE para variável explicativa - Número de alunos [Fonte: ITE, 2003]

\begin{tabular}{|c|c|c|c|c|}
\hline Dados & & Dias da $S$ & ina Típico & \\
\hline $\begin{array}{c}N^{o} \text { de estudos } \\
\text { catalogados }\end{array}$ & 51 & 34 & 62 & 62 \\
\hline Variável Explicativa $(X)$ & $\mathrm{N}^{\circ}$ de Estudantes & $\mathrm{N}^{\circ}$ de Estudantes & $\mathrm{N}^{\circ}$ de Estudantes & $\mathrm{N}^{\circ}$ de Estudantes \\
\hline$X$ médio & 1382 & 1282 & 1290 & 1290 \\
\hline Período de Análise & Dia & $\begin{array}{l}\text { Hora no pico do } \\
\text { Tráfego da Tarde } \\
\text { (16 às 18hs) }\end{array}$ & $\begin{array}{l}\text { Hora no pico da manhã do } \\
\text { empreendimento }\end{array}$ & $\begin{array}{c}\text { Hora no pico da tar- } \\
\text { de do empreendi- } \\
\text { mento }\end{array}$ \\
\hline $\begin{array}{l}\text { Taxa média de viagens } \\
\text { de automóveis por } \\
\text { unidade de tempo }\end{array}$ & 1,71 & 0,14 & 0,41 & 0,28 \\
\hline $\begin{array}{l}\text { Intervalo da Taxa e } \\
\text { Desvio Padrão }\end{array}$ & $\begin{array}{c}0,71-3,96 / \text { dia } \\
(1,49)\end{array}$ & $\begin{array}{c}0,03-0,38 / \text { hora } \\
(0,38)\end{array}$ & $\begin{array}{c}0,14-1,15 / \text { hora } \\
(0,67)\end{array}$ & $\begin{array}{c}0,10-0,74 / \text { hora } \\
(0,54)\end{array}$ \\
\hline $\begin{array}{l}\text { Variação entre fluxos } \\
\text { de entradas e saídas }\end{array}$ & $50 \%$ entrando e $50 \%$ saindo & $\begin{array}{l}47 \% \text { entrando e } \\
53 \% \text { saindo }\end{array}$ & $\begin{array}{c}69 \% \text { entrando e } \\
31 \% \text { saindo }\end{array}$ & $\begin{array}{c}32 \% \text { entrando e } \\
8 \% \text { saindo }\end{array}$ \\
\hline Equação & $\ln (T)=0,81 \cdot \ln (X)+1,86$ & NI* & $\ln (T)=0,77 \cdot \ln (X)+0,69$ & NI* \\
\hline$R^{2}$ & 0,56 & NI* & NI* & NI* \\
\hline
\end{tabular}

Tabela 2. Resultados ITE para variável explicativa - Número de empregados [Fonte: ITE, 2003]

\begin{tabular}{|c|c|c|c|c|}
\hline \multirow{2}{*}{$\begin{array}{c}\text { Dados } \\
N^{o} \text { de estudos } \\
\text { catalogados }\end{array}$} & \multicolumn{4}{|c|}{ Dias da Semana Típico } \\
\hline & 51 & 24 & 52 & 52 \\
\hline Variável Explicativa $(X)$ & $\mathrm{N}^{\circ}$ de Empregados & $\mathrm{N}^{\circ}$ de Empregados & $\mathrm{N}^{\circ}$ de Empregados & $\mathrm{N}^{\circ}$ de Empregados \\
\hline$X$ médio & 119 & 136 & 119 & 119 \\
\hline Período de Análise & Dia & $\begin{array}{l}\text { Hora no pico do } \\
\text { Tráfego da Tarde } \\
\text { (16 às } 18 \mathrm{hs})\end{array}$ & $\begin{array}{l}\text { Hora no pico da } \\
\text { manhã do empreen- } \\
\text { dimento }\end{array}$ & $\begin{array}{l}\text { Hora no pico da tarde do } \\
\text { empreendimento }\end{array}$ \\
\hline $\begin{array}{l}\text { Taxa média de viagens } \\
\text { de automóveis por } \\
\text { unidade de tempo }\end{array}$ & 19,74 & 1,55 & 4,63 & 3,21 \\
\hline $\begin{array}{c}\text { Intervalo da Taxa e } \\
\text { Desvio Padrão }\end{array}$ & $\begin{array}{c}4,28-35,26 / \text { dia } \\
(7,95)\end{array}$ & $\begin{array}{c}0,41-3,00 / \text { hora } \\
(1,39)\end{array}$ & $\begin{array}{c}0,54-10,20 / \text { hora } \\
(2,84)\end{array}$ & $\begin{array}{c}1,13-6,98 / \text { hora } \\
(2,06)\end{array}$ \\
\hline $\begin{array}{l}\text { Variação entre fluxos } \\
\text { de entradas e saídas }\end{array}$ & $\begin{array}{c}50 \% \text { entrando e } \\
50 \% \text { saindo }\end{array}$ & $\begin{array}{l}54 \% \text { entrando e } \\
46 \% \text { saindo }\end{array}$ & $\begin{array}{l}71 \% \text { entrando e } \\
29 \% \text { saindo }\end{array}$ & $\begin{array}{l}31 \% \text { entrando e } \\
69 \% \text { saindo }\end{array}$ \\
\hline Equação & $\ln (T)=1,13 \cdot \ln (X)+2,31$ & NI* & NI* & NI* \\
\hline$R^{2}$ & 0,56 & NI* & NI* & NI* \\
\hline
\end{tabular}

(NI*: não informado)

serão apresentadas neste estudo, para efeito de comparação (fraco movimento de final de semana nas IEs e grande variabilidade na razão número de estudantes e a área construída).

A metodologia utilizada nos estudos do ITE considera apenas o modo automóvel particular na obtenção de taxas e modelos, apesar de citar a pesquisa de outros modos (ITE, 2008). Prevê, igualmente, a realização de contagens, preferencialmente por contadores automáticos, junto a acessos exclusivos dos empreendimentos. As viagens atraídas e produzidas em cada período de contagem são categorizadas como primárias (base domiciliar), desviadas e de passagem. Essa categorização é realizada com base em questionários ou entrevistas aplicadas a amostras de usuários, paralelamente às contagens.
Mais recentemente modelos computacionais têm sido desenvolvidos no Reino Unido, na Nova Zelândia e pelo próprio ITE, automatizando banco de dados sobre viagens ligadas aos tipos de atividades nos novos empreendimentos. Um dos ganhos desta automatização é permitir a análise de composições de diferentes usos num mesmo empreendimento (multiple use), e o acúmulo de contagens (manuais classificadas, automáticas direcionais de tráfego, multi-modais) e entrevistas complementares.

No Brasil, apenas a Companhia de Engenharia de Tráfego de São Paulo, CET - SP (CET, 1983; CET, 2000), propôs modelos e taxas para Instituições de Ensino Médio, e apenas para a cidade de São Paulo. Nesses estudos as características de divisão modal diversificada e o uso compartilhado de alunos de dife- 
Tabela 3. Resultados para Instituições de Ensino Médio [Fonte: adaptado de CET-SP, 2000]

\begin{tabular}{|c|c|c|c|c|c|c|c|c|c|}
\hline & & & Per & agem de & unos por $n$ & do de viag & $(\%)$ & & \\
\hline$P e$ & & A nó & Auto 1 & Auto 2 & Coletivo & Coletivo & Outros & Total & por auto \\
\hline Manhã & Entrada & 17 & 37 & 32 & 10 & 2 & 2 & 100 & 1,54 \\
\hline IVIanta & Saída & 20 & 23 & 21 & 30 & 5 & 1 & 100 & 1,73 \\
\hline Tarde & Entrada & 18 & 37 & 35 & 9 & 1 & 0 & 100 & 1,50 \\
\hline 1 artuc & Saída & 16 & 31 & 25 & 27 & 1 & 0 & 100 & 1,58 \\
\hline Inteoral & Entrada & 3 & 46 & 45 & 0 & 6 & 0 & 100 & 1,55 \\
\hline & Saída & 3 & 46 & 41 & 6 & 4 & 0 & 100 & 1,57 \\
\hline
\end{tabular}

rentes graus no mesmo veículo já aparecem como características importantes nas escolas estudadas.

O estudo de 1983 foi realizado de forma agregada entre escolas de diversos graus de ensino, sem diferenciação de categoria de IEM, pública ou privada, sendo elaborados os modelos mostrados nas Equações 1 a 3 , que utilizam como variáveis de estudo, o número de alunos, a área total de salas de aula, em metros quadrados, e o número de salas de aula, respectivamente (CET-SP, 1983).

$$
\begin{gathered}
V=0,432 \cdot N A-106,303 \\
V=0,343 \cdot A S+434,251 \\
V=22,066 \cdot N S+102,186
\end{gathered}
$$

em que,
$V$ : estimativa do número médio de viagens atra- ídas pelo PGV na hora-pico;
$N A$ : número total de alunos;
$A S$ : área total de salas de aula $\left(\mathrm{m}^{2}\right)$;
NS: número de salas de aula.

O modelo apresentado na Equação 1 possui $\mathrm{R}^{2}$ igual a 0,707 e, de acordo com a CET, só deve ser usado para PGVs com NA $<13.000$ alunos. Já o modelo da Equação 2 apresenta $R^{2}$ de 0,684 , devendo ser utilizado somente nos casos em que AS $<13.000 \mathrm{~m}^{2}$. Finalmente, o modelo da Equação 3 , cujo $\mathrm{R}^{2}$ é igual a 0,850 , deve ser empregado quando (NS/NA) $\geq 0,005$.

Em 2000, um novo estudo apresentou resultados para IEMs nos turnos da manhã, tarde e em turno integral, não distinguindo entre IEMs públicas e privadas, e continua estudando apenas as viagens de alunos, não de todos os usuários das IEMs (CET-SP, 2000). Esses resultados, entretanto, são significativos como indicativos da escolha modal: mais de $70 \%$ dos alunos pesquisados afirmam usar o automóvel particular como meio de transporte. A Tabela 3 resume alguns dos principais resultados desse estudo.

\section{METODOLOGIA PARA ESTUDO DA GERAÇÃO DE VIAGENS EM IEMS}

A metodologia adotada no estudo é a apresentada na Figura 1, e suas principais etapas são descritas a seguir. Os instrumentos e o próprio procedimento adotado para a coleta de dados foram definidos a partir dos trabalhos de Jacques et al. (2006) e Souza (2007). Nestes estudos, pesquisadores da Universidade de Brasília, apoiados por pesquisadores da REDPGV (http://redpgv.coppe.ufrj.br/), desenvolveram procedimento de coleta e tratamento de dados baseado na aplicação de questionários para a análise do comportamento médio das viagens dos usuários típicos de Instituições de Ensino.

\subsection{Seleção da amostra e definição dos períodos de pesquisa}

O número inicial de IEMs a serem pesquisadas foi baseado na metodologia do ITE (2001), que propõe que a pesquisa seja realizada com no mínimo cinco estabelecimentos para o desenvolvimento de modelos de geração de viagens. O uso de questionários, ao invés do uso de contadores veiculares automáticos, é apoiado em recomendações do ITE (2001) para as condições de matriz modal diversificada, e também em experiência do Departamento de Transportes do Reino Unido (DfT, 2007).

No caso das IEMs brasileiras, cada turno de funcionamento das Instituições de Ensino Médio pesquisadas representa uma população diferente atendida, operando cada turno como uma IEM diferente, e ampliando a número inicial de IEMs pesquisadas. Os períodos de pesquisa típicos foram referenciados, para efeito da geração de viagens, ao início e término de cada turno. Para a presente aplicação, pequenas adaptações foram realizadas nos questionários aplicados para facilitar a diferenciação de turnos e a compilação de dados. 


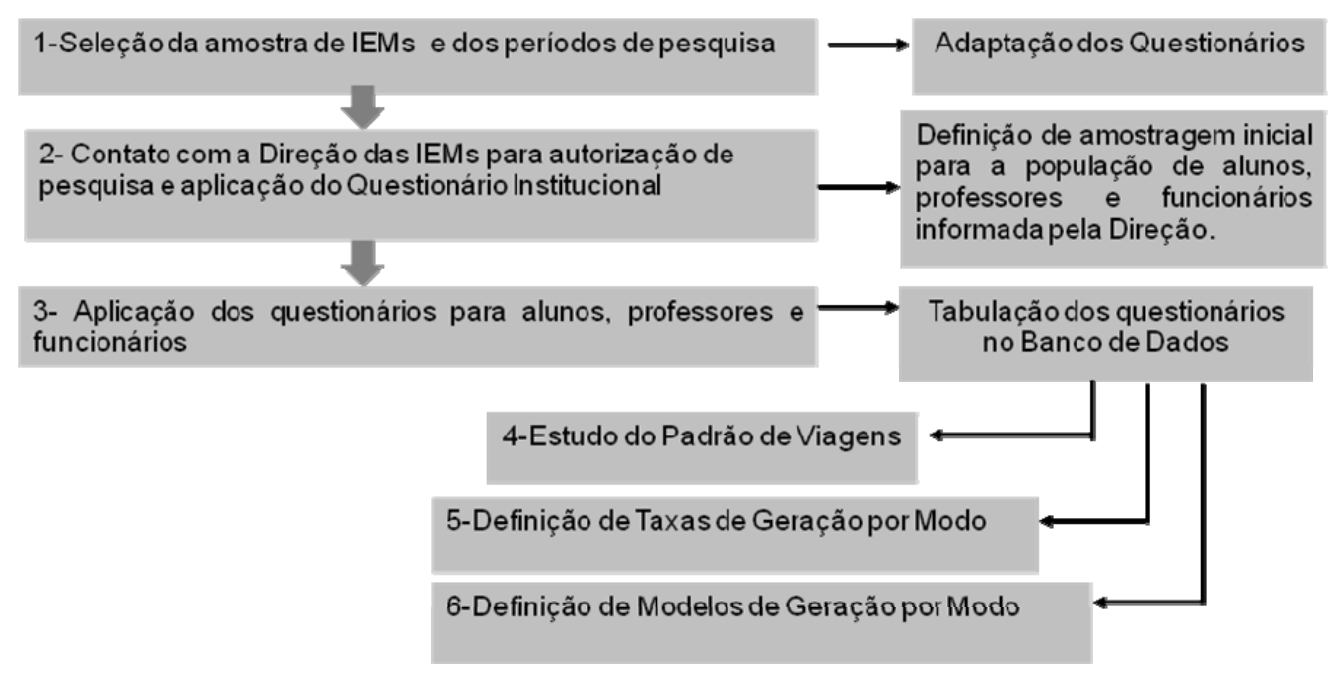

Figura 1. Metodologia adotada para o estudo de geração de viagens em IEMs

\subsection{Contato com a direção das IEMs}

Este contato visa obter a autorização necessária da direção de cada IEM para a realização da coleta de dados na instituição. É importante destacar que, como a metodologia prevê a coleta de dados junto a professores e funcionários e a aplicação de questionário aos alunos em sala de aula, a autorização e cooperação da direção da escola é imprescindível para a realização da pesquisa. O questionário onde são coletados os dados da instituição deve ser preenchido durante esse contato com a direção. A definição do número mínimo de questionários a ser aplicado em cada instituição é feita a partir dos dados do número de frequentadores do estabelecimento por turno, do nível de significância desejado, e do erro máximo aceitável para as estimativas. Esses dados permitem, também, que, dado o número de questionários respondidos, se determine o erro máximo a priori da estimativa.

\subsection{Aplicação dos questionários}

A forma de aplicação dos questionários, sobretudo aos alunos, deve ser discutida com a direção da escola. Preferencialmente, devem-se marcar alguns horários para essa aplicação em sala de aula, procurando cobrir, na medida do possível, todas as turmas. Durante essas idas à escola também devem ser aplicados os questionários aos professores e funcionários. No caso desses últimos, para interferir o mínimo na sua rotina de trabalho, o questionário pode ser aplicado na forma de entrevista, o que agiliza o seu preenchimento. Em geral, não é possível atingir toda a população de usuários do estabelecimento. Assim, deve-se pelo menos garantir que o número de questionários respondidos assegure um erro de estimativa aceitável para o nível de significância definido, e que a amostra seja formada aleatoriamente, sem qualquer viés. Posteriormente, os dados coletados são organizados e tratados, na forma descrita por Bertazzo (2008).

\subsection{Estudo do padrão de viagens}

O estudo do padrão de viagens é necessário para a melhor definição e interpretação das taxas e modelos de estimação das viagens geradas, a serem elaborados a partir dos dados coletados. Para efeito desse estudo, foram analisadas as mesmas características das viagens estudadas por Souza (2007), que são: período de realização, origem e destino, modo de transporte, principais vias utilizadas, e locais de embarque e desembarque.

\subsection{Procedimento na elaboração de taxas e modelos de geração de viagens}

Devem ser elaboradas Taxas de Geração de Viagens e Modelos para estimar as viagens atraídas e produzidas apenas para os turnos que cumpriram os critérios estabelecidos na metodologia do ITE (2001) em termos do número de observações mínimas necessárias. A partir do estudo do padrão de viagens, devem ser definidas as taxas e modelos por turno, para os modos de transporte mais utilizados, e, em separado também, para os dois tipos de IEMs (pública e privada). No caso do modo automóvel particular, como o questionário permite saber o número de pessoas que utilizaram o mesmo veículo para realizar a viagem, as taxas e modelos devem ser construídas em termos do número de viagens veiculares. Já para os demais modos (a pé, por onibus, etc.) as taxas e modelos são elaboradas considerando as viagens de pessoas por cada modo. Na elaboração das taxas e modelos, devem ser consideradas diferentes variáveis independentes vinculadas às características populacionais e à estrutura física das IEMs estudadas, selecionadas a partir das comumente adotadas em estudos de instituições de ensino como PGV.

Para a definição das Taxas de Geração de Viagens, deve ser determinado o valor médio e o correspondente intervalo de confiança (IC) para um nível de signi- 
ficância $(\alpha)$ desejado, o qual permite verificar se a taxa média é significativamente diferente de zero. Para a elaboração dos modelos, recomenda-se analisar, previamente, os diagramas de dispersão entre a variável dependente e cada uma das variáveis explicativas, de modo a facilitar a identificação dos modelos lineares e não lineares a serem testados. Além da significância estatística dos parâmetros do modelo, devem ser verificados o coeficiente de determinação associado $\left(\mathrm{R}^{2}\right)$ e o correspondente diagrama de resíduos. Seguindo a mesma abordagem do ITE, as taxas e modelos devem ser determinadas para o total de viagens, considerando todos os usuários da instituição.

\section{ESTUDO DE CASO}

As etapas metodológicas descritas na seção 4 foram aplicadas para o estudo das taxas e modelos de geração de viagens de Brasília. Os resultados completos dessa aplicação são apresentados em Bertazzo (2008). Destaca-se, entre eles, que a análise do padrão de viagens apontou a necessidade de se incluirem nos estudos de geração de viagens para IEMs localizadas em Brasília, outros modos de transporte, além do automóvel particular.

\subsection{Seleção da amostra e aplicação dos questionários}

Para a constituição da amostra de IEMs, foram considerados os dados de matrícula nas IEMs no Distrito Federal, públicas e privadas (BRASIL, 2006), e a localidade da Regional de Ensino do Plano Piloto e Cruzeiro como limitação geográfica. Apesar de várias IEMs terem sido contatadas, conforme o procedimen-

Tabela 4. Seleção IEMs Públicas

\begin{tabular}{|c|c|c|c|c|c|c|c|c|c|c|c|c|c|}
\hline IEM & \multicolumn{4}{|c|}{ Dados populacionais } & \multicolumn{4}{|c|}{ Dados amostrais } & \multicolumn{5}{|c|}{ Dados da infra-estrutura } \\
\hline Usuário & \multicolumn{3}{|c|}{ Alunos } & \multirow{2}{*}{$\begin{array}{c}\begin{array}{c}\text { Profs. } \\
e \\
\text { funcs. }\end{array} \\
\text { Dia }\end{array}$} & \multicolumn{3}{|c|}{ Alunos } & \multirow{2}{*}{$\begin{array}{c}\begin{array}{c}\text { Profs. } \\
e \\
\text { funcs. }\end{array} \\
\text { Dia }\end{array}$} & \multirow{2}{*}{$\begin{array}{c}\begin{array}{c}\text { Área } \\
\text { terreno }\end{array} \\
m^{2}\end{array}$} & \multirow{2}{*}{$\begin{array}{c}\begin{array}{c}\text { Área } \\
\text { constr. } \\
m^{2}\end{array}\end{array}$} & \multirow{2}{*}{$\begin{array}{c}\text { Salas } \\
\text { de } \\
\text { aula } \\
\text { un. }\end{array}$} & \multirow{2}{*}{$\begin{array}{c}\text { vagas } \\
\text { auto } \\
\text { un. }\end{array}$} & \multirow{2}{*}{$\begin{array}{c}\text { vagas } \\
\text { bicicl. } \\
\text { un. }\end{array}$} \\
\hline Turno & $M$ & $T$ & $N$ & & $M$ & $T$ & $N$ & & & & & & \\
\hline $\begin{array}{c}\text { IEM-PU } \\
01\end{array}$ & 540 & 256 & 525 & 113 & 179 & - & 263 & 55 & 110.000 & 6.250 & 20 & 152 & 22 \\
\hline $\begin{array}{c}\text { IEM-PU } \\
02\end{array}$ & 296 & 0 & 410 & 56 & 142 & - & 154 & 31 & 32.500 & 3.100 & 12 & 70 & 23 \\
\hline $\begin{array}{c}\text { IEM-PU } \\
03\end{array}$ & 359 & 0 & 183 & 39 & 233 & - & 115 & 17 & 64.660 & 2.280 & 10 & 82 & 30 \\
\hline $\begin{array}{c}\text { IEM-PU } \\
04\end{array}$ & 779 & 473 & 239 & 101 & 149 & - & 165 & 30 & 80.000 & 4.879 & 24 & 234 & 10 \\
\hline $\begin{array}{c}\text { IEM-PU } \\
05\end{array}$ & 545 & 421 & 0 & 99 & 198 & 142 & - & 27 & 23.000 & 3.083 & 14 & 70 & 10 \\
\hline Média & 503,8 & 230,0 & 271,4 & 81,6 & 180,2 & 142,0 & 174,3 & 32,0 & $57.540,0$ & 3.918 & 14 & 94 & 21 \\
\hline $\begin{array}{l}\text { Desvio } \\
\text { padrão }\end{array}$ & 189,0 & 224,7 & 203,7 & 32,2 & 37,2 & 63,5 & 95,1 & 14,0 & $42.636,4$ & 1.613 & 7 & 54 & 12 \\
\hline
\end{tabular}

Tabela 5. Seleção IEMs Privadas

\begin{tabular}{|c|c|c|c|c|c|c|c|c|c|c|c|c|c|}
\hline IEM & \multicolumn{4}{|c|}{ Dados populacionais } & \multicolumn{4}{|c|}{ Dados amostrais } & \multicolumn{5}{|c|}{ Dados da infra-estrutura } \\
\hline Usuário & \multicolumn{3}{|c|}{ Alunos } & \multirow{2}{*}{$\begin{array}{c}\begin{array}{c}\text { Profs. } \\
\boldsymbol{e}\end{array} \\
\text { funcs. }\end{array}$} & \multicolumn{3}{|c|}{ Alunos } & \multirow{2}{*}{$\begin{array}{c}\begin{array}{c}\text { Profs. } \\
e \\
\text { funcs. }\end{array} \\
\text { Dia }\end{array}$} & \multirow{2}{*}{$\begin{array}{c}\begin{array}{c}\text { Área } \\
\text { terreno }\end{array} \\
m^{2}\end{array}$} & \multirow{2}{*}{$\begin{array}{c}\text { Área } \\
\text { constr. } \\
m^{2}\end{array}$} & \multirow{2}{*}{$\begin{array}{c}\text { Salas } \\
\text { de } \\
\text { aula } \\
\text { un. }\end{array}$} & \multirow{2}{*}{$\begin{array}{c}\text { vagas } \\
\text { auto } \\
\text { un. }\end{array}$} & \multirow{2}{*}{$\begin{array}{r}\text { vagas } \\
\text { bicicl } \\
\text { un. }\end{array}$} \\
\hline Turno & $M$ & $T$ & $N$ & & $M$ & $T$ & $N$ & & & & & & \\
\hline $\begin{array}{c}\text { IEM-PR } \\
01\end{array}$ & 414 & 0 & 0 & 63 & 175 & - & - & 11 & 10.350 & 3.832 & 50 & 37 & 0 \\
\hline $\begin{array}{c}\text { IEM-PR } \\
02\end{array}$ & 272 & 0 & 0 & 107 & 185 & - & - & 19 & 16.340 & 2.606 & 27 & 24 & 0 \\
\hline $\begin{array}{c}\text { IEM-PR } \\
03\end{array}$ & 345 & 0 & 0 & 54 & 192 & - & - & 27 & 7.150 & 4.015 & 34 & 28 & 15 \\
\hline $\begin{array}{c}\text { IEM-PR } \\
04\end{array}$ & 280 & 0 & 0 & 135 & 245 & - & - & 44 & 15.000 & 12.000 & 42 & 135 & 10 \\
\hline $\begin{array}{c}\text { IEM-PR } \\
05\end{array}$ & 597 & 445 & 0 & 122 & 175 & 129 & - & 61 & 15.000 & 8.051 & 32 & 70 & 0 \\
\hline Média & 381,6 & 89,0 & 0,0 & 96,2 & 194,4 & 129,0 & 0,0 & 32,4 & $12.768,0$ & 6.101 & 37 & 59 & 5 \\
\hline $\begin{array}{l}\text { Desvio } \\
\text { padrão }\end{array}$ & 133,3 & 199,0 & 0,0 & 36,0 & 29,2 & 57,7 & 0,0 & 20,1 & $3.877,2$ & 3.883 & 9 & 46 & 7 \\
\hline
\end{tabular}


to apresentado na seção 4.2, a amostra foi composta por 05 IEMs públicas, de um total de 13 instituições deste tipo na área de estudo, e por 05 IEMs privadas, selecionadas entre as 35 instituições privadas que ofertam ensino médio na área estudada.

Conforme previsto, embora se tenha tentado aplicar o questionário da pesquisa a todos os alunos, professores e funcionários da escola, o número de questionários respondidos por cada categoria nas diferentes IEMs não atingiu esta meta, mas ainda assim possibilitou uma margem de erro máxima de 5\%. A composição da amostra por categoria de usuários por IEM é apresentado nas Tabelas 4 e 5, juntamente com os dados de cada instituição.

De acordo com decisão específica de cada estabelecimento, diversas foram as formas de aplicação dos questionários aos alunos, entre as quais em aulas de reforço, no início do período de aula ou acompanhando a rotina de um professor específico em diversas turmas.

Em função dos dados obtidos, as taxas e modelos só foram determinados para os turnos da manhã e da noite, para as IEMs públicas, e para o turno da manhã para as IEMs privadas.

\subsection{Taxas e modelos de geração de viagens obtidos}

O ITE (2001) recomenda que não sejam usadas as suas taxas, e sim criadas novas taxas, quando as taxas locais diferirem em pelo menos $15 \%$ das taxas recomendadas. Para avaliação da aplicabilidade das taxas do ITE (2003) para viagens veiculares, às IEMs estudadas, as taxas do ITE foram ajustadas para entrada e saída e comparadas com as taxas obtidas. Os valores informados pelo ITE (2003) em cada período de contagem (ver Tabelas 1 e 2) foram referenciados aos períodos de entrada e saída das IEMs pesquisadas: valores $69 \%$ entrando, $68 \%$ saindo para taxas do ITE para alunos; e $71 \%$ entrando e $69 \%$ saindo para professores e funcionários. A Tabela 6 apresenta os resultados que permitem a verificação de que o uso das taxas do ITE para as instituições estudadas não atende ao critério de diferença aceitável estabelecido pela instituição. A única exceção é para taxa de viagens produzidas em IEMs privadas no turno da manhã.

$\mathrm{Na}$ Tabela 7, são apresentadas todas as taxas médias de geração de viagens obtidas no estudo, com os correspondentes intervalos para um nível de confiança igual a 95\% mostrados entre parênteses. Essas taxas referem-se, no caso das viagens veiculares, ao número total de automóveis que chegam (atraídos) e saem do empreendimento (produzidos) por período pesquisado, enquanto que as taxas de viagens por ônibus e a pé referem-se ao número total de usuários regulares que se deslocam por meio desses modos. Nos dois casos, as taxas mostradas na Tabela 7 referem-se ao total de viagens, relacionado ao número de alunos e ao número de professores e funcionários. Entre os modelos de geração de viagens (atração e produção) obtidos no estudo, os apresentados na Tabela 8 são os que apresentam os melhores valores para o coeficiente de correlação $\left(\mathrm{R}^{2}\right)$, além de coeficientes das variáveis explicativas estatisticamente significativas, para nível de significância de 5\% (exceto nos dois casos indicados na tabela).

Tabela 6. Avaliação da aplicabilidade das taxas ITE para as IMEs estudadas

\begin{tabular}{|c|c|c|c|c|}
\hline Estudo & $\begin{array}{c}\text { Atração } \\
\text { Taxa média *1 }\end{array}$ & $\begin{array}{c}\text { Comparação } \\
\text { Tx obtida e } \\
\text { Tx ITE } *^{5}\end{array}$ & $\begin{array}{c}\text { Produção } \\
\text { Taxa média } *^{2}\end{array}$ & $\begin{array}{c}\text { Comparação } \\
\text { Tx obtida e } \\
\text { Tx ITE }{ }^{* 5}\end{array}$ \\
\hline \multicolumn{5}{|c|}{ Para variável Número de Alunos } \\
\hline ITE & $0,41 \cdot 69 \%=0,283$ & - & $0,28 \cdot 68 \%=0,19$ & - \\
\hline IEM PU Manhã & 0,100 & $-65 \%$ & 0,053 & $-72 \%$ \\
\hline IEM PU Noite & ND & - & 0,083 & $-56 \%$ \\
\hline IEM PR Manhã & 0,347 & $23 \%$ & 0,172 & $-10 \%$ \\
\hline \multicolumn{5}{|c|}{ Para variável Número de Professores e funcionários ${ }^{* 4}$} \\
\hline ITE & $4,63 \cdot 71 \%=3,27$ & - & $3,21 \cdot 69 \%=2,277$ & - \\
\hline IEM PU Manhã & 0,569 & $-83 \%$ & 0,310 & $-86 \%$ \\
\hline IEM PU Noite & $\mathrm{ND}^{* 3}$ & - & 0,383 & $-83 \%$ \\
\hline IEM PR Manhã & 1,491 & $-55 \%$ & 0,674 & $-70 \%$ \\
\hline $\begin{array}{l}*^{1} \text { Taxa do ITE para } h \\
*^{2} \text { Taxas do ITE para } \\
*^{3} \text { ND não definida }-1 \\
*^{4} \text { No caso do ITE “en } \\
*^{5} \frac{\text { Taxa }_{\text {obtida }}-\text { Taxa }}{\text { Taxa }} \text { ITE }\end{array}$ & $\begin{array}{l}\text { co da manhã do empree } \\
\text { pico da tarde do empre } \\
\text { ados estatisticamente nã } \\
\text { ees"; }\end{array}$ & $\begin{array}{l}\text { to; } \\
\text { nto; } \\
\text { icativos; }\end{array}$ & & \\
\hline
\end{tabular}


A partir dos resultados obtidos, verifica-se que a hipótese inicial do trabalho de diferenciação entre IEMs públicas e privadas não se confirmou em todos os turnos e modos estudados, mas apenas em algumas situações. São elas: (i) taxas por número de alunos para viagens veiculares atração e produção manhã; (ii) taxas por número de professores e funcionários para viagens veiculares atração e produção manhã; (iii) taxas por número de alunos e por número de professores e funcionários para viagens a pé na produção manhã; (iv) nos modelos globais que incluem a variável dummy relativa ao caráter da IEM pública ou privada (D2) para viagens veiculares, por ônibus e a pé.

Como mostram as Tabelas 7 e 8 , além das taxas e modelos desenvolvidos para a estimativa da geração de viagens por modo de transporte e categoria de IEM, para cada turno considerado no estudo, foram desenvolvidos alguns elementos globais por modo (atração e/ou produção), agregando todos os turnos. Os modelos globais, incluindo a variável Dummy relativa aos turnos (D1) confirmam a diferenciação da geração de viagens por turno.

\section{CONCLUSÕES}

O estudo de geração de viagens, propósito deste trabalho, é o primeiro estágio para o desenvolvimento de estudos de impacto de implantação de empreendimentos tipo PGV, como é o caso das IEMs nos sistemas viários e de transportes.

Tomando como referência a metodologia do ITE, foi elaborada uma metodologia alternativa para este tipo de estudo e mais compatível com a realidade das IEMs públicas e privadas brasileiras. A aplicação da metodologia às IEMs localizadas em Brasília permitiu a determinação de taxas e modelos significativos, para um nível de significância de $5 \%$.

Os resultados revelaram a importância da consideração de diferentes modos, variação da geração de viagens entre os turnos (manhã e noite) e de tipo de IEMs considerados (público e privado). Eles mostraram, também, que as taxas médias informadas pelo ITE para as viagens veiculares em situações semelhantes às estudadas não são aplicáveis em Brasília, nem devem ser usadas indiscriminadamente quando se tratar da realidade brasileira. Para futuros trabalhos, recomenda-se a consulta a resultados mais recentes do ITE (2008), ampliando a análise.

Tabela 7. Taxas de gerações de viagens

\begin{tabular}{|c|c|c|c|c|c|c|c|c|}
\hline & \multicolumn{4}{|c|}{ Taxas na atração } & \multicolumn{4}{|c|}{ Taxas na produção } \\
\hline & \multicolumn{2}{|c|}{ PorNA } & \multicolumn{2}{|c|}{ Por NPF } & \multicolumn{2}{|c|}{ Por NA } & \multicolumn{2}{|c|}{ Por NPF } \\
\hline & Individual & Agregada & Individual & Agregada & Individual & Agregada & Individual & Agregada \\
\hline \multicolumn{9}{|c|}{ VIAGENS VEICULARES } \\
\hline IEMs PU - manhã & $\begin{array}{c}\mathbf{0 , 1 0 0} \\
(0,026 ; 0,174) \\
\end{array}$ & \multirow{2}{*}{$\begin{array}{c}\mathbf{0 , 0 8 3} \\
(0,043 ; 0,123)\end{array}$} & $\begin{array}{c}\mathbf{0 , 5 6 9} \\
(0,205 ; 0,932) \\
\end{array}$ & \multirow{3}{*}{$\begin{array}{c}\mathbf{0 , 8 1 9} \\
(0,456 ; 1,181)\end{array}$} & $\begin{array}{c}\mathbf{0 , 0 5 3} \\
(0,020 ; 0,085) \\
\end{array}$ & \multirow{3}{*}{$\begin{array}{c}\mathbf{0 , 0 6 6} \\
(0,039 ; 0,094)\end{array}$} & $\begin{array}{c}\mathbf{0 , 3 1 0} \\
(0,183 ; 0,437) \\
\end{array}$ & \multirow{3}{*}{$\begin{array}{c}\mathbf{0 , 3 4 2} \\
(0,220 ; 0,463)\end{array}$} \\
\hline IEMs PU - noite & ND & & ND & & \begin{tabular}{c|}
$\mathbf{0 , 0 8 3}$ \\
$(0,008 ; 0,158)$ \\
\end{tabular} & & $\begin{array}{c}\mathbf{0 , 3 8 3} \\
(0,013 ; 0,753) \\
\end{array}$ & \\
\hline IEMs PR - manhã & $\begin{array}{c}\mathbf{0 , 3 4 7} \\
(0,220 ; 0,474) \\
\end{array}$ & & $\begin{array}{c}\mathbf{1 , 4 9 1} \\
(0,631 ; 2,350) \\
\end{array}$ & & \begin{tabular}{c|c|}
$\mathbf{0 , 1 7 2}$ \\
$(0,100 ; 0,244)$ \\
\end{tabular} & & $\begin{array}{c}\mathbf{0 , 6 7 4} \\
(0,518 ; 0,829) \\
\end{array}$ & \\
\hline \multicolumn{9}{|c|}{ VIAGENS POR ÔNIBUS } \\
\hline IEMs PU - manhã & $\begin{array}{c}\mathbf{0 , 6 4 1} \\
(0,385 ; 0,896) \\
\end{array}$ & \multirow{3}{*}{$\begin{array}{c}\mathbf{0 , 4 5 0} \\
(0,333 ; 0,567)\end{array}$} & $\begin{array}{c}\mathbf{4 , 3 9 8} \\
(1,706 ; 7,087) \\
\end{array}$ & \multirow{3}{*}{$\begin{array}{c}2,568 \\
(1,472 ; 3,664)\end{array}$} & $\begin{array}{c}\mathbf{0 , 3 4 2} \\
(0,062 ; 0,623) \\
\end{array}$ & \multirow{3}{*}{$\begin{array}{c}\mathbf{0 , 2 8 5} \\
(0,178 ; 0,392)\end{array}$} & ND & \multirow{3}{*}{$\begin{array}{c}\mathbf{1 , 5 6 0} \\
(0,725 ; 2,395)\end{array}$} \\
\hline IEMs PU -noite & $\begin{array}{c}\mathbf{0 , 3 1 0} \\
(0,144 ; 0,476) \\
\end{array}$ & & $\begin{array}{c}\mathbf{1 , 3 5 5} \\
(0,589 ; 2,122) \\
\end{array}$ & & \begin{tabular}{c|}
$\mathbf{0 , 3 6 4}$ \\
$(0,171 ; 0,557)$ \\
\end{tabular} & & $\begin{array}{c}\mathbf{1 , 5 3 2} \\
(0,921 ; 2,143) \\
\end{array}$ & \\
\hline IEMs PR - manhã & $\begin{array}{c}\mathbf{0 , 3 7 2} \\
(0,191 ; 0,553) \\
\end{array}$ & & $\begin{array}{c}\mathbf{1 , 7 0 7} \\
(0,488 ; 2,927) \\
\end{array}$ & & ND & & ND & \\
\hline \multicolumn{9}{|l|}{ VIAGENS A PÉ } \\
\hline IEMs PU -manhã & $\begin{array}{c}\mathbf{0 , 2 1 1} \\
(0,011 ; 0,411)\end{array}$ & \multirow{3}{*}{$\begin{array}{c}\mathbf{0 , 2 4 8} \\
(0,159 ; 0,337)\end{array}$} & $\begin{array}{c}\mathbf{1 , 2 1 8} \\
(0,154 ; 2,282)\end{array}$ & \multirow{2}{*}{$\begin{array}{c}\mathbf{1 , 5 6 6} \\
(0,909 ; 2,224)\end{array}$} & ND & \multirow{2}{*}{$\begin{array}{c}\mathbf{0 , 2 8 1} \\
(0,156 ; 0,405)\end{array}$} & ND & \multirow{2}{*}{$\begin{array}{c}\mathbf{1 , 3 9 2} \\
(0,719 ; 2,064)\end{array}$} \\
\hline IEMs PU - noite & $\begin{array}{c}\mathbf{0 , 4 2 7} \\
(0,310 ; 0,544) \\
\end{array}$ & & $\begin{array}{c}2, \mathbf{0 0 2} \\
(0,663 ; 3,340) \\
\end{array}$ & & $\begin{array}{c}\mathbf{0 , 4 3 1} \\
(0,317 ; 0,545) \\
\end{array}$ & & $\begin{array}{c}\mathbf{2 , 0 2 3} \\
(0,668 ; 3,378) \\
\end{array}$ & \\
\hline IEMs PR - manhã & $\begin{array}{c}\mathbf{0 , 1 4 2} \\
(0,051 ; 0,233) \\
\end{array}$ & & $\begin{array}{c}\mathbf{0 , 5 8 9} \\
(0,283 ; 0,895) \\
\end{array}$ & & \begin{tabular}{c|}
$\mathbf{0 , 0 7 2}$ \\
$(0,026 ; 0,118)$ \\
\end{tabular} & & $\begin{array}{c}\mathbf{0 , 2 8 2} \\
(0,172 ; 0,392) \\
\end{array}$ & \\
\hline
\end{tabular}

Legenda:

PU - pública; PR - privada; NA - núm ero de alunos; NPF - número de professores e fun cionários; ND - não definida (estatisticamente não significativa para $\alpha=5 \%$ ) 


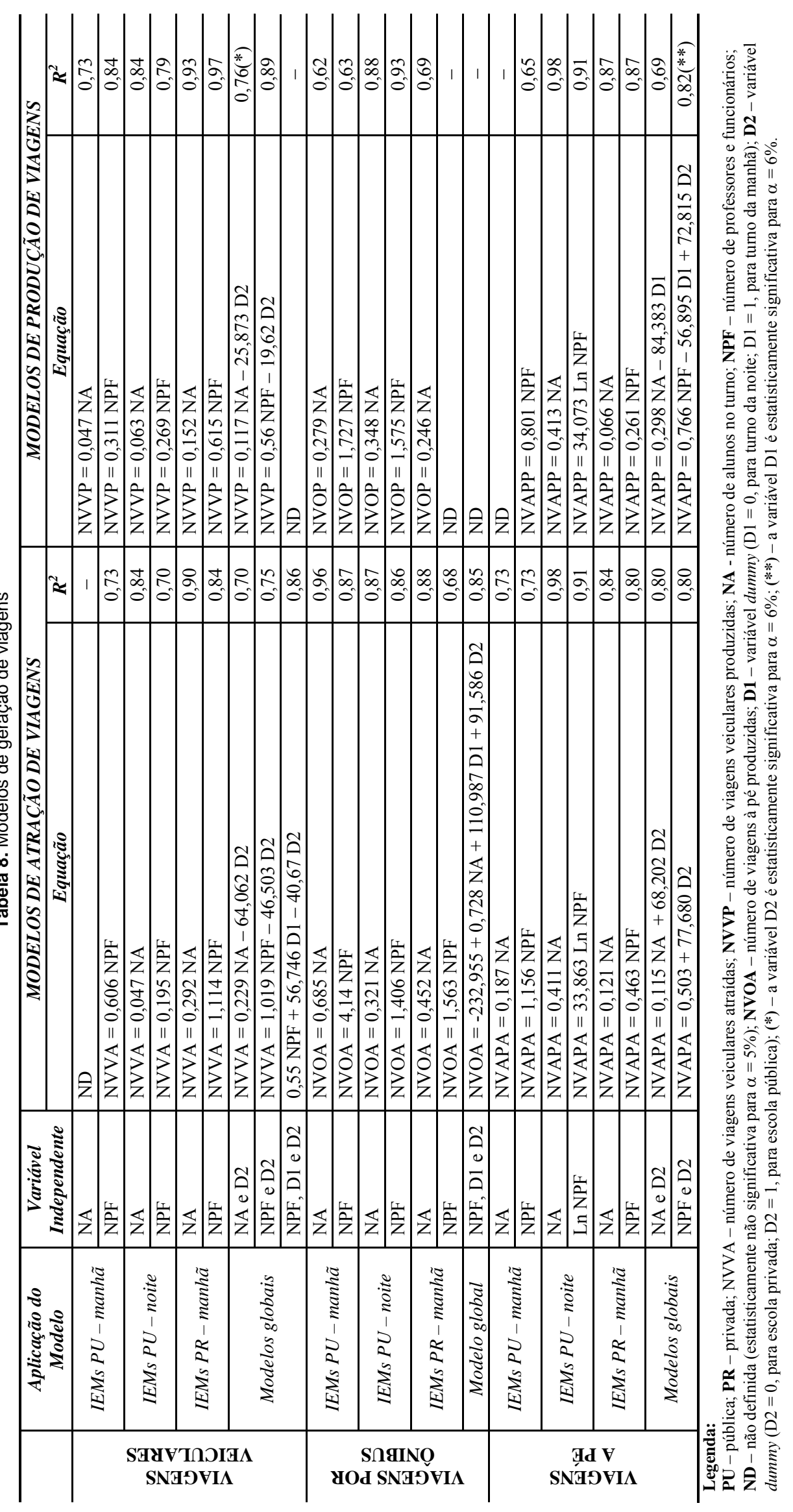




\section{REFERÊNCIAS BIBLIOGRÁFICAS}

Bertazzo, A. B. S. Estimativa e Avaliação do Padrão de Viagens Geradas para Instituições de Ensino Médio. Dissertação de Mestrado. Universidade de Brasília, DF, 2008. 198p.

Brasil. Ministério da Educação. Instituto Nacional de Estudos e Pesquisas Educacionais Anísio Teixeira. Resultados do Censo Escolar 2005. Brasília, 2006. Disponível em <http://www.mec.gov.br/ > consultado em 30/08/2006.

CET-SP. Boletim Técnico n. 32.Polos geradores de tráfego. São Paulo, 1983.

Boletim Técnico 36. Polos Geradores de Tráfego II. 2ed. São Paulo: CET, 2000.

Departamento Nacional de Trânsito. Manual brasileiro de sinalização de trânsito do DENATRAN: sinalização de áreas escolares. Brasília,DF, DENATRAN, 2000.

Department for Transport and Communities and Local Government Guidance on Transport Assessment. London: TSO. 2007. Disponível em

$<$ http://www.dft.gov.uk/162259/165237/202657/guidanceontapdf $>$, consultado em 29/03/2007.

Ferreira, D. C.Comparação do Padrão de Viagens em Escolas de Ensino Médio Pública e Privada: um estudo de caso. Monografia de Projeto Final de Transportes. Universidade de Brasília. Faculdade de Tecnologia. Departamento de Engenharia Civil e Ambiental. Brasília: UnB, 2007.

ITE - Institute of Transportation Engineers. Trip Generation Handbook an ITE Recommended Practice. Washington, DC, 2001.

Trip Generation. Washington, DC: ITE.2003.

Trip Generation. Washington, DC: ITE.2008

Jacques, M.A.P. et al. Procedimento para a coleta e registro dos dados obtidos junto a instituições de ensino no contexto de Pólos Geradores de Viagens. In. Anais do XX Congresso da Associação Nacional de Ensino e Pesquisa em Transportes. Brasília: ANPET. 2006.

Nunes, J. L. Estudo da Demanda por estacionamento em Instituições de Ensino Superior. Dissertação de mestrado - Faculdade de Tecnologia, Universidade de Brasília, DF, 2005.

Portugal, L. S; Goldner, L. G.. Estudo de polos geradores de tráfego e seus impactos nos sistemas viários e de transportes, Ed. Edgard Blücher Ltda, São Paulo, 2003.

Souza, S. C. F.. Modelos para Estimativa de Viagens Geradas por Instituições de Ensino Superior. Dissertação de Mestrado, Publicação T.DM009A/2007, Departamento de Engenharia Civil e Ambiental, Faculdade de Tecnologia, Universidade de Brasília, DF, 2007. 181p. 